ARTICLE

\title{
CRISPR-based genome editing in primary human pancreatic islet cells
}

Romina J. Bevacqua ${ }^{1}$, Xiaoqing Dai ${ }^{2}$, Jonathan Y. Lam (1) ${ }^{1}$, Xueying Gu', Mollie S. H. Friedlander ${ }^{1}$, Krissie Tellez ${ }^{1}$, Irene Miguel-Escalada (10 3,4, Silvia Bonàs-Guarch (10 3,4, Goutham Atla ${ }^{3,4}$, Weichen Zhao', Seung Hyun Kim', Antonia A. Dominguez 5,6 , Lei S. Qi (10) 5,6,7, Jorge Ferrer (1) 3,8,4, Patrick E. MacDonald ${ }^{2}$ \& Seung K. Kim (1) 1,9,10,11凶

Gene targeting studies in primary human islets could advance our understanding of mechanisms driving diabetes pathogenesis. Here, we demonstrate successful genome editing in primary human islets using clustered regularly interspaced short palindromic repeats (CRISPR) and CRISPR-associated protein 9 (Cas9). CRISPR-based targeting efficiently mutated protein-coding exons, resulting in acute loss of islet $\beta$-cell regulators, like the transcription factor PDX1 and the $\mathrm{K}_{\text {ATP }}$ channel subunit KIR6.2, accompanied by impaired $\beta$ cell regulation and function. CRISPR targeting of non-coding DNA harboring type 2 diabetes (T2D) risk variants revealed changes in ABCC8, SIX2 and SIX3 expression, and impaired $\beta$-cell function, thereby linking regulatory elements in these target genes to T2D genetic susceptibility. Advances here establish a paradigm for genetic studies in human islet cells, and reveal regulatory and genetic mechanisms linking non-coding variants to human diabetes risk.

\footnotetext{
${ }^{1}$ Department of Developmental Biology, Stanford University School of Medicine, Stanford, CA, USA. ${ }^{2}$ Alberta Diabetes Institute and Department of Pharmacology, University of Alberta, Edmonton, AB, Canada. ${ }^{3}$ Bioinformatics and Genomics Program, Centre for Genomic Regulation (CRG), The Barcelona Institute of Science and Technology (BIST), Barcelona, Spain. ${ }^{4}$ Centro de Investigación Biomédica en Red de Diabetes y Enfermedades Metabólicas Asociadas (CIBERDEM), Barcelona, Spain. ${ }^{5}$ Department of Bioengineering, Stanford University, Stanford, CA, USA. ${ }^{6}$ Department of Chemical and Systems Biology, Stanford University, Stanford, CA, USA. ${ }^{7}$ Chem-H, Stanford University, Stanford, CA, USA. ${ }^{8}$ Section of Genetics and Genomics, Imperial College London, London, UK. ${ }^{9}$ Department of Medicine (Endocrinology), Stanford University School of Medicine, Stanford, CA, USA. ${ }^{10}$ Northern California JDRF Center of Excellence, Stanford University School of Medicine, Stanford, CA, USA. ${ }^{11}$ Stanford Diabetes Research Center, Stanford University School of Medicine, Stanford, CA, USA. ${ }^{凶}$ email: seungkim@stanford.edu
} 
ancreatic islets of Langerhans are crucial endocrine organs that regulate internal metabolic homeostasis, and diseases like diabetes mellitus have been linked to impaired function or loss of islet cells, especially insulin-secreting $\beta$-cells. Prior studies $^{1-7}$ have unveiled the genetic origins of islet cell dysfunction or loss in all forms of diabetes, including familial, type 1 (T1D), type 2 (T2D), and type $3 c$ diabetes. Genetic susceptibility in $\mathrm{T} 2 \mathrm{D}$ and $\mathrm{T} 1 \mathrm{D}$ is driven by non-coding variants enriched in active islet enhancers ${ }^{5,6,8}$. Thus, experimental systems for modeling and modulating regulatory variants or elements in human islet cells could define molecular determinants of diabetes susceptibility. However, gene regulation differs substantially between human islets and experimental animal or islet-like cells produced from human cell lines 9 . For example, genes encoding the transcription factors SIX2 and SIX3 are expressed in adult human $\beta$ cells, but not in rodent $\beta$-cells ${ }^{10,11}$, highlighting the need for genetics in primary human islets.

Site-specific gene editing ${ }^{12,13}$ enabled by CRISPR, and CRISPR-associated protein 9 (Cas9) or targeted gene activation ${ }^{14}$ (CRISPRa) mediated by nuclease-deactivated Cas9 (dCas9), could transform our understanding of genetic mechanisms governing vital tissue and organ functions and disease development. However, it remains unknown if these modern genome editing tools can be applied to native human pancreatic islet cells: CRISPRbased gene editing or control of gene expression in primary human islet cells has not previously been reported. This reflects unmet challenges to achieve efficient, durable delivery of Cas9 and guide RNA (sgRNA) to intact islets, including the sensitivity of islet cells to methods used to achieve this delivery ${ }^{15}$. Furthermore, CRISPR-Cas9-based DNA editing may be less efficient in quiescent cells, like primary adult human islet cells, which are post-mitotic $^{16-18}$ and not amenable to the selection of desired gene modifications.

Recent studies have used a strategy based on the dispersion of pancreatic islets and reaggregation into primary organoids (called pseudoislets) to enable RNA knock down or misexpression ${ }^{10,19-22}$. Pseudoislets are able to reconstitute native intercellular interactions of islet cells in a multicellular state that recapitulates islet cyto-architecture and function ${ }^{10,20-22}$. However, to date, genetic approaches in human pseudoislets have not allowed direct modification of $\beta$-cell DNA, including potential regulatory elements in non-coding DNA.

Here we describe the use of CRISPR/Cas9 and CRISPR/dCas9based enhancer activation (CRISPRa) in primary human islets. Delivery and expression of sgRNAs and Cas9 permitted efficient genome editing, resulting in targeting of protein-coding exons and non-coding regulatory elements in primary human islet cells, loss of gene expression, and impairment of cellular function, assessed in vitro and in transplanted pseudoislets. By targeting regulatory elements with sgRNAs and dCas9 linked to the activation domains VP64-p65-Rta ${ }^{23}$, we also induced the expression of genes from endogenous genomic sequences of human islet cells. These experiments revealed an essential function of PDX1 in primary mature human islet $\beta$-cells, as anticipated from mouse and human genetics, and established functional target genes of regulatory elements that carry $\mathrm{T} 2 \mathrm{D}$-associated variants. Thus, our findings demonstrate the feasibility of manipulating coding and non-coding regulatory genomic regions in primary human islets, and thereby expand the experimental repertoire for dissecting genetic mechanisms underlying human diabetes.

\section{Results}

CRISPR/Cas9 targeting of $P D X 1$ protein-coding sequence in primary human islets. To achieve site-specific gene editing in primary adult human islet cells, we used a strategy of islet dispersion and lentiviral transduction, followed by reaggregation and culture of islet cell clusters in pseudoislets for 6 days (Fig. 1A, Methods; ${ }^{10,20}$ ). Use of an "all-in-one" lentivirus permitted simultaneous expression of sgRNA and Cas 9 to target a protein-coding sequence in exon 1 of $P D X 1$, and production of Green Fluorescent Protein from a GFP transgene to mark infected cells (Fig. 1B). Infection with a lenticonstruct encoding a non-targeting sgRNA, Cas9, and GFP served as a control (CRISPR-Control). Islet cell transduction efficiency, determined by flow cytometry counting of $\mathrm{GFP}^{+}$cells, averaged $41 \%$ (Supplementary Fig. 1A-C). We chose to target PDX1, which encodes a transcription factor previously shown to be crucial for $\beta$ cell function in mouse islets, and which is mutated in human diabetes $^{3,24-26}$. After transducing PDX1 sgRNAs, we isolated GFP ${ }^{+}$ human islet cells 6 days after infection, and performed TIDE PCR and digital droplet (dd)PCR (Methods; ${ }^{27-29}$ ) with gene-specific probes to genomic DNA (Fig. 1C, D; $n=3$ independent donors; Supplementary Fig. 2A, B). Insertion-deletion mutations (indels) were detected in an average of $66 \%$ of sequences by TIDE PCR; Fig. 2C, D) and in $48 \%$ of sequences by ddPCR (Supplementary Fig. 2A, B). Immunostaining studies revealed $72 \%$ reduction of $\mathrm{GFP}^{+}$ $\mathrm{PDX}^{+}$cells (Fig. $1 \mathrm{E}-\mathrm{I} ; n=3$ independent donors); thus, extensive loss of PDX1 protein in primary human islet cells corroborated our molecular findings. To assess targeting specificity, we used an in silico approach $^{30}$ to identify the likeliest genomic sites for "off-target" modification using CRISPR/Cas9 and the sgRNA targeting PDX1 exon 1. Sequencing analysis revealed that indels were undetectable in 7/7 predicted genomic off-target sites (Supplementary Fig. 2C, D). Thus, exposure of $\beta$-cells to CRISPR/Cas9 for several days in cultured pseudoislets achieved substantial, specific targeting in PDX1 exon 1.

Loss-of-function phenotypes in primary human islets from CRISPR/Cas9 targeting of PDX1. After CRISPR/Cas9 targeting of $P D X 1$, we found that expression of $\beta$-cell-specific genes previously identified as direct targets of $P d x 1$ regulation in mouse studies $26,31,32$ was also impaired in $\mathrm{GFP}^{+}$human islet cells (Methods). qRT-PCR revealed a significant reduction of mRNAs encoding PDX1 and the $\beta$-cell genes INSULIN, MAFA, IAPP, and SLC2A2 (Fig. 2A, B; $n=4$ independent donors). Total insulin content of the CRISPR-PDX1 purified $\mathrm{GFP}^{+}$cells was reduced by $60 \%$ compared to controls (Fig. 2G; $n=3$ independent donors) and total insulin content of the CRISPR-PDX1 intact pseudoislets was reduced by $40 \%$ (Supplementary Fig. $3 \mathrm{~A}-\mathrm{E}$; $n=4$ independent donors). We also noted an increased average expression of a-cell transcripts and glucagon content (Fig. 2B, Supplementary Fig. 3F-J; $n=4$ independent donors), although these did not reach statistical significance. Direct PDX1 targets like $M A F A$ and $S L C 2 A 2$ are regulators of $\beta$-cell function ${ }^{33}$, including stimulussecretion coupling, and whole-cell patch-clamping revealed electrophysiological defects in $\beta$-cells after CRISPR/Cas 9 targeting of PDX1, including impaired $\mathrm{Ca}^{2+}$ and $\mathrm{Na}^{+}$currents, and reduced glucose-stimulated $\beta$-cell exocytosis (Fig. $2 \mathrm{C}-\mathrm{F} ; n=3$ independent donors). In vitro perifusion assays 5 days after lentiviral infection revealed reduced glucose-dependent insulin secretion by CRISPR-PDX1 pseudoislets compared to controls (Fig. 2H-I; $n=3$ independent donors: Methods). Together, these data revealed that CRISPR/Cas9-induced PDX1 loss led to impaired physiological function in native human $\beta$-cells.

To evaluate the durability of defects and the impact of PDX1 targeting on in vivo function, we transplanted CRISPR-PDX1 and control pseudoislets into immunocompromised, non-diabetic NSG mice ( $n=3$ independent donors; Methods) and assessed glucosestimulated insulin secretion. Four weeks after transplantation, intraperitoneal glucose tolerance tests (IPGTT) revealed $>70 \%$ reduction of human insulin secretion from pseudoislets with PDX1 loss compared to controls (Fig. 2J: $n=4$ mice for CRISPR-PDX1 or 
A

Primary Human Islets

\section{$\underset{\text { Day } 0}{\stackrel{\text { Disperse }}{\longrightarrow}}$}

lentiviral

transduction

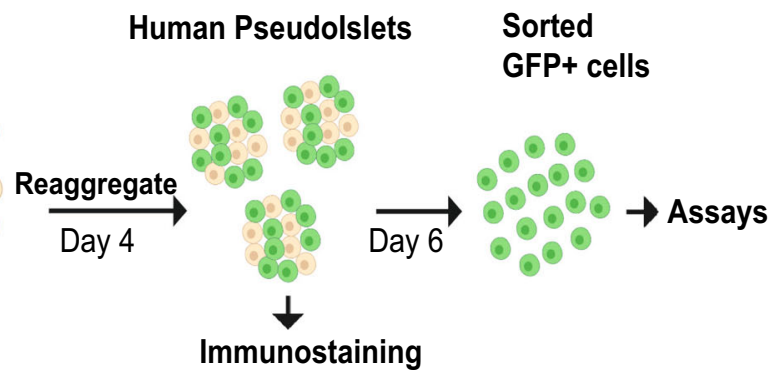

B

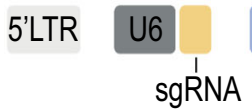

C

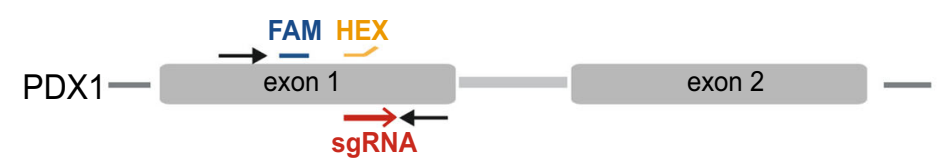

$C A T G G G T C C T T G T A A A G C T \quad T G C G G G G \subset C C \subset N A A G A$

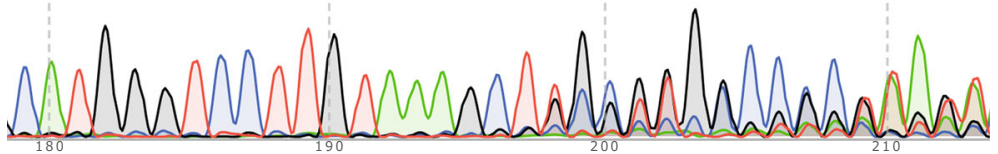

D
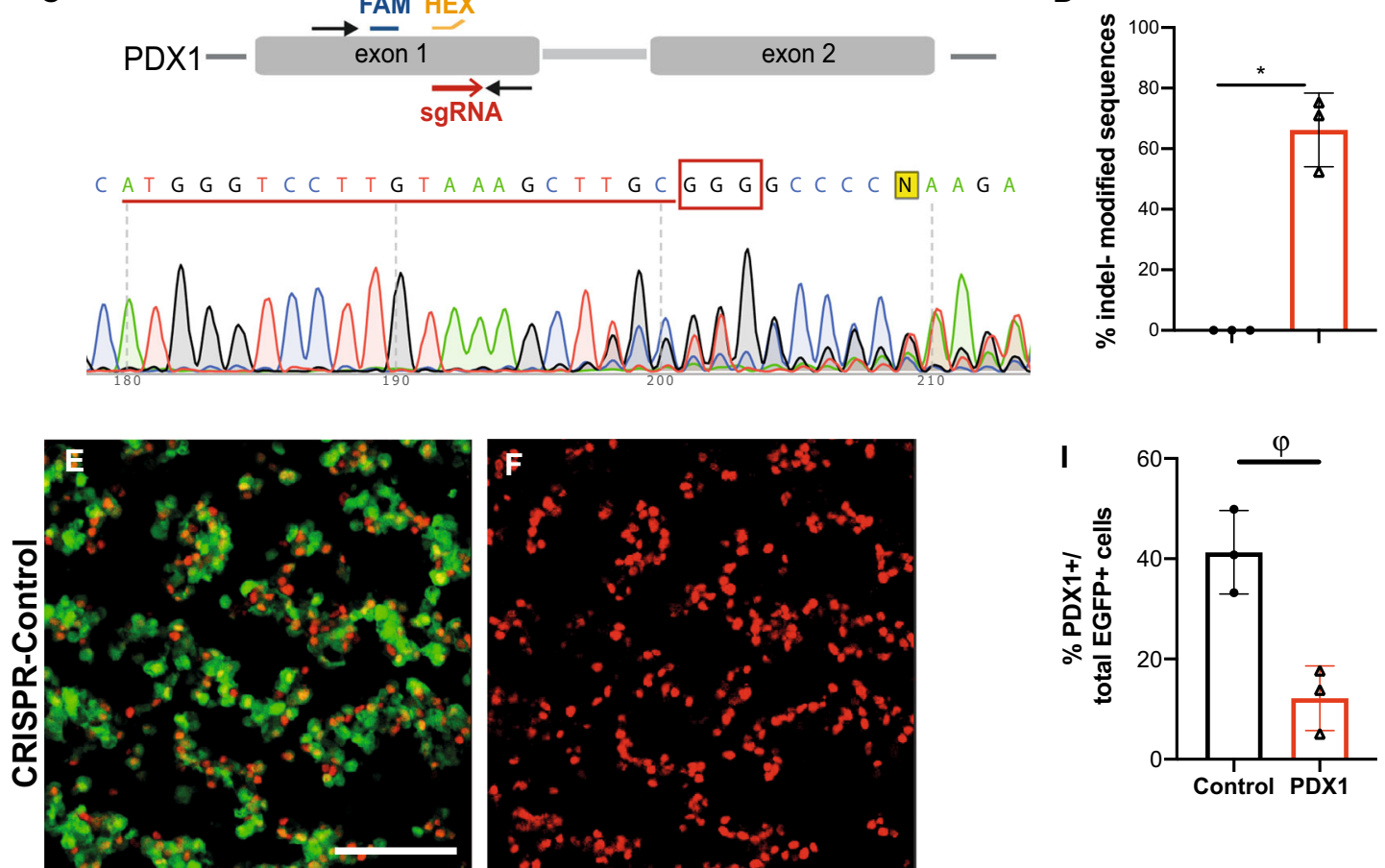
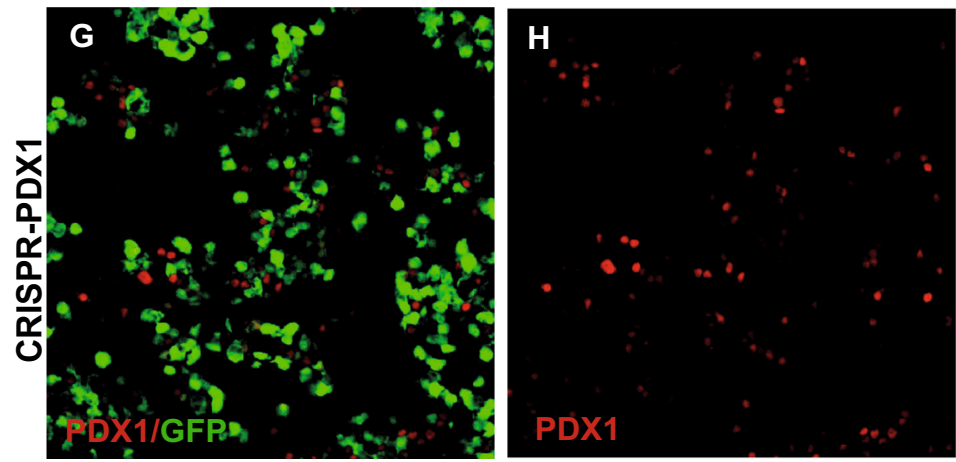

Fig. 1 CRISPR/Cas9 targeting of PDX1 protein-coding sequence in primary human islets. A Schematics of the human pseudoislet system. B Scheme of the lentiCRISPR construct used. C PDX1 sequence, showing the sgRNA sequence (underlined in red, PAM sequence recognized by Cas9 in red box). FAM (blue) and HEX (orange) probes used for ddPCR. D Percentage of indel-modified sequences detected by the TIDE algorithm ( $P=0.01)$. E-H Immunostaining of CRISPR-Control versus CRISPR-PDX1, showing GFP+ PDX1+ cells ( $n=3$ independent donor repetitions); scale bar: $50 \mu \mathrm{m}$. I Quantification of GFP+ PDX $1^{+}$cells for CRISPR-Control versus CRISPR-PDX1 $P=0.07 ; n=3$ independent donor repetitions). Data are presented as mean values \pm SD. Two-tailed $t$ tests were used to generate $P$-values. ${ }^{\star} P<0.05, \phi P<0.1$. Source data are provided as a Source Data file. 

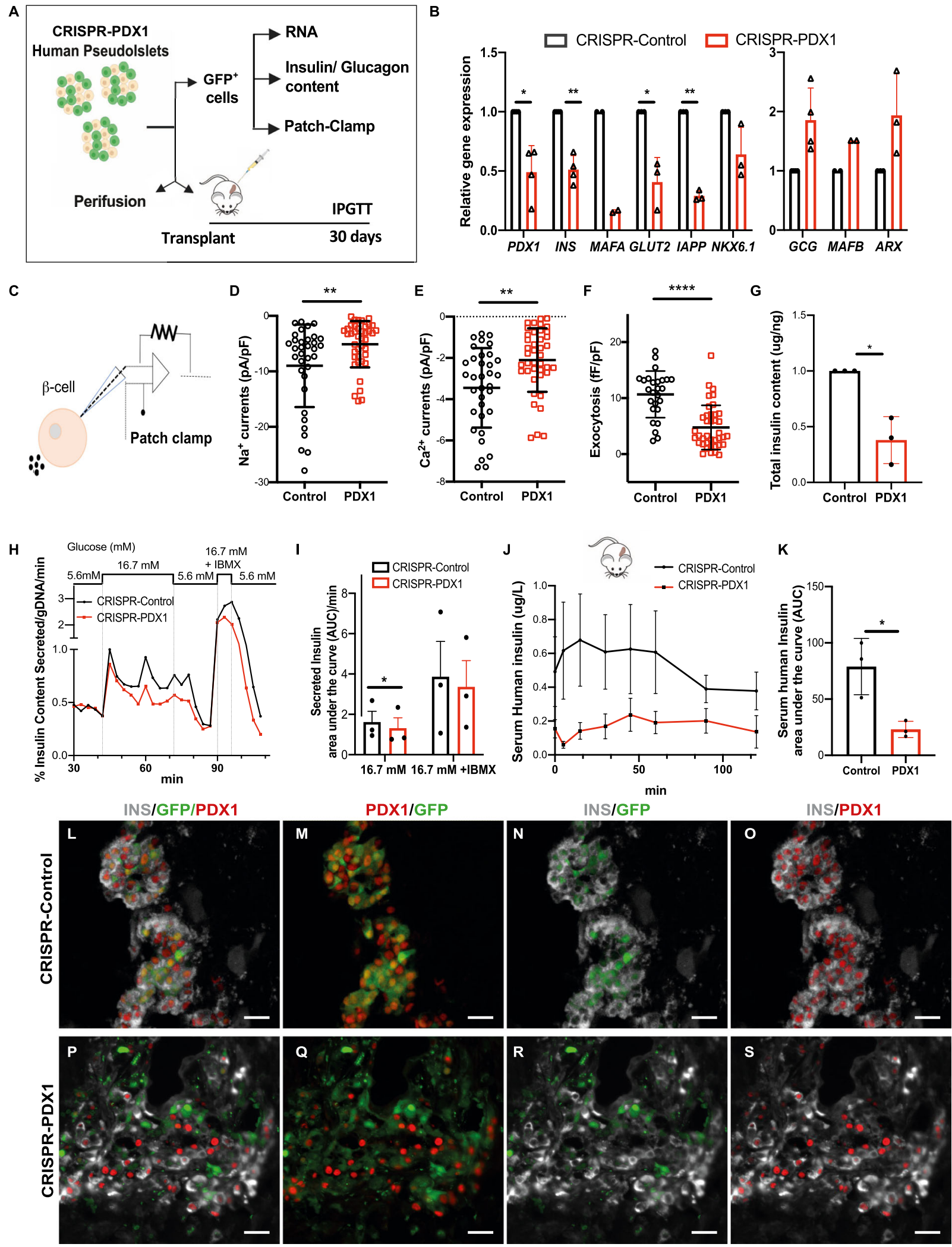

for controls), and a corresponding significant reduction in human insulin output for the CRISPR-PDX1 condition (Fig. 2K: area under the curve, AUC: $P<0.05$; Methods). Serum glucose levels after IPGTT were indistinguishable in mice transplanted with CRISPRPDX1 or control pseudoislets (Supplementary Fig. 3K-L), likely reflecting intact host islet function. Compared to control grafts, immunostaining of grafts with PDX1 targeting showed reduced
PDX1 and INS production in $\mathrm{GFP}^{+}$islet $\beta$-cells (Fig. 2P-S; Supplementary Fig. 3M, N). Together, these experiments indicate that the lentiCRISPR-pseudoislet system can be efficiently applied to primary human islet cells, providing evidence in mature human islets that PDX1 targeting and loss leads to durable impairment of hallmark $\beta$-cell phenotypes, including cardinal physiological $\beta$-cell functions. 
Fig. 2 CRISPR/Cas9 targeting of PDX1 in primary human islets leads to loss-of-function phenotypes. A Schematics of analyses performed to characterize the phenotype of CRISPR-PDX1 human pseudoislets. B Relative gene expression of known PDX1-targets in CRISPR-PDX1 GFP+ cells relative to CRISPR-Control GFP+ cells ( $P=0.0199$ for PDX1, $P=0.037$ for INS, $P=0.0386$ for GLUT2, $P=0.013$ for IAPP). C Scheme of $\beta$-cell patch clamp. D-F $\beta$ cells show electrophysiological defects after CRISPR/Cas9 targeting of PDX1, including $(\mathbf{D})$ impaired $\mathrm{Na}^{+}(P=0.057)$ and $(\mathbf{E}) \mathrm{Ca}{ }^{2+}$ currents $(P=0.0012)$, and $(\mathbf{F})$ reduced glucose-dependent $\beta$-cell exocytosis $(P<0.0001)$. G Total insulin content in CRISPR-PDX1 GFP ${ }^{+}$cells relative to CRISPR-Control GFP ${ }^{+}$ cells $(P=0.036)$. H Secreted human insulin by CRISPR-Control versus CRISPR-PDX1 pseudoislets in vitro, following perifusion with media containing Glucose at 5.6, 6.7, and $16.7 \mathrm{mM}+$ IBMX. I Area under the curve (AUC) of secreted human insulin by CRISPR-Control versus CRISPR-PDX1 pseudoislets at $16.7 \mathrm{mM}(P=0.0387)$ and $16.7 \mathrm{mM}$ Glucose + IBMX. J Serum human insulin levels in the blood of NSG mice one-month following transplantation of CRISPR-PDX1 versus CRISPR-Control pseudoislets, following intraperitoneal glucose tolerance tests (IPGTT) ( $n=3$ independent human islets donors). K Area under the curve of the serum human insulin released by transplanted CRISPR-PDX1 versus CRISPR-Control pseudoislets, shown in $(\mathbf{J})(P=0.049)$. K-S Immunostaining of grafts recovered one-month following transplantation with CRISPR-Control (K-O) versus CRISPR-PDX1 (P-S) pseudoislets; INS: gray, GFP: green, PDX1: red. Scale bars: 20 um. ( $n=3$ independent donor repetitions). Data are presented as mean values \pm SD for (B-G, K) and as mean \pm SEM for $(\mathbf{I})$ and $(\mathbf{J})$. Two-tailed t tests were used to generate $P$-values. ${ }^{\star} P<0.05,{ }^{\star \star} P<0.01,{ }^{\star \star \star \star} P<0.0001$. Source data are provided as a Source Data file.
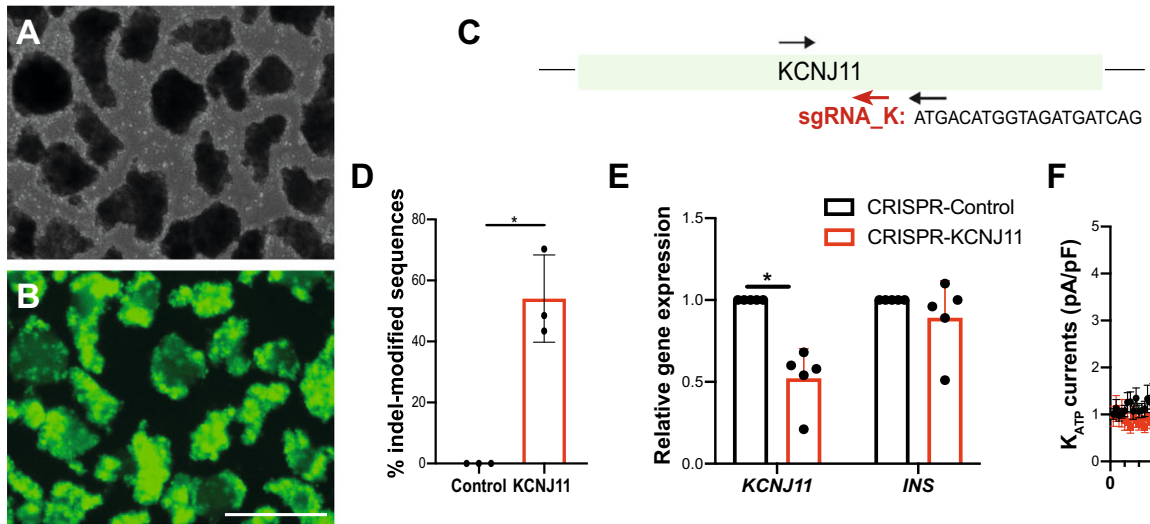

$\mathbf{E}$

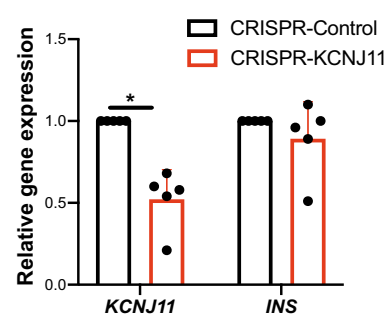

$\mathbf{F}$

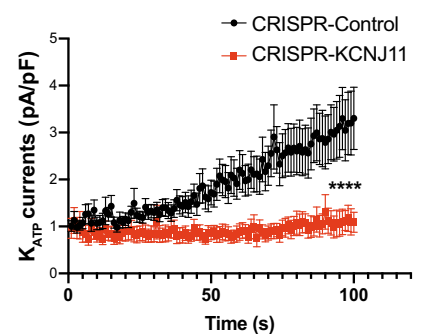

Fig. 3 CRISPR/Cas9 targeting of KCNJ11 protein-coding sequence in primary human islets. A, B Human pseudoislets 5 days post-infection with CRISPRKCNJ11 lentiviruses (A) bright field, (B) blue light ( $488 \mathrm{~nm}$ ), scale bar: $500 \mu \mathrm{m}$ ( $n=3$ independent donors). C Schematics of KCNJ11 sequence, showing the sgRNA sequence (sgRNA_K, red). Arrows indicate primers used for PCR. D Percentage of indel-modified sequences detected by TIDE algorithm ( $n=3$ independent donors; $P=0.0225)$. E qRT-PCR of GFP+ cells, CRISPR-KCNJ11 (red), CRISPR-Control (Black) $(n=5$ independent donors; $P=0.0175)$.

F Patch clamping in single $\beta$-cells: measurement of $K_{\text {ATP }}$ currents in CRISPR-KCNJ11 ( $n=19$ cells, 3 replicates) and CRISPR-Control ( $n=25,3$ replicates) $(P<0.0001)$. Data are presented as mean values \pm SD for $\left(\mathbf{D}\right.$-E) and mean values \pm SEM for $(\mathbf{F})$. Two-tailed t tests were used to generate $P$-values. ${ }^{\star} P<$ $0.05,{ }^{\star \star \star \star} P<0.0001$. Source data are provided as a Source Data file.

CRISPR/Cas9 targeting of $K C N J 11$ protein-coding sequence in primary human islets. To assess the general use of CRISPR/Cas9 gene targeting in primary human islets, we built additional lentiCRISPR constructs to target exon 1 of KCNJ11, which encodes the KIR6.2 pore-forming subunit of $\mathrm{K}_{\mathrm{ATP}}$ channels (Fig. 3C: Methods). Six days after transduction, we sorted $\mathrm{GFP}^{+}$cells from pseudoislets (Fig. 3A, B), and used TIDE to quantify that an average $54 \%$ of sequences were indel-modified (Fig. $3 \mathrm{D} ; n=3$ independent donors). qRT-PCR of $\mathrm{GFP}^{+}$cells demonstrated a 52\% KIR6.2 mRNA reduction; by contrast, INS mRNA levels were not altered (Fig. 3E; $n=5$ independent donors). Sequencing analysis of the likeliest genomic off-target sites (Methods; ${ }^{30}$ ) revealed that indels were undetectable in $6 / 6$ of these sites (Supplementary Fig. 4). To assess if KCNJ11 targeting was sufficient to impair $\mathrm{K}_{\mathrm{ATP}}$ channels function, we used patch clamping in single $\beta$-cells, and confirmed that $\mathrm{K}_{\mathrm{ATP}}$ currents were severely reduced after CRISPR-KCNJ11 (Fig. 3F; $n=3$ independent donors). Thus, CRISPR/Cas9 gene targeting can be used at distinct genetic loci to achieve loss-of-function in human $\beta$-cells.

CRISPR/Cas9 targeting of cis-regulatory genomic regions in human islet cells. The vast preponderance of genetic risk for diabetes mellitus maps to non-coding $\mathrm{DNA}^{34}$, and it is known that the function of regulatory elements is extremely dependent on cell-type and on the stage of islet cell differentiation. Thus, functional studies of non-coding genomic regions in native human islets would be a major advance for islet biology and diabetes research, and we investigated if our lentiCRISPRpseudoislet method could generate targeted deletions in postulated gene regulatory regions. The KCNJ11-ABCC8 locus harbors non-coding variants linked to diabetes risk ${ }^{1,34-38}$. For example, the variant rs1002226 falls within an enhancer hub in the KCNJ11-ABCC8 locus associated with diabetes risk, and we adapted our lentiCRISPR construct to simultaneously express two sgRNAs (sg_EK1 and sg_EK2) and induce a small deletion in the putative enhancer encompassing this risk SNP (Fig. 4A, B: Methods; $\left.{ }^{35,39}\right)$. In purified $\mathrm{GFP}^{+}$cells, qRT-PCR analysis revealed a reduction of $A B C C 8$ mRNA, while KCNJ11 mRNA was not significantly changed (Fig. $4 \mathrm{C} ; n=4$ independent donors). This finding corresponds well with data from our prior chromatin looping $\mathrm{pc}-\mathrm{HiC}$ studies $^{35}$ revealing an interaction linking the putative enhancer region harboring rs1002226 to $A B C C 8$, but not to KCNJ11 (Fig. 4B). Moreover, the mRNA levels of USH1C and NCR3LG1, two neighboring genes located in the same topologically associated domain (TAD) as this putative enhancer ${ }^{35}$, were not altered (Fig. 4C; $n=3$ independent donors); likewise, INS mRNA levels did not significantly change (Fig. 4C; $n=4$ independent donors). Consistent with these findings, patch clamping of single $\beta$-cells revealed a reduction in $\mathrm{K}_{\mathrm{ATP}}$ currents following targeting of the enhancer region flanking rs1002226 in the KCNJ11-ABCC8 locus (Fig. 4D; $n=3$ independent donors). Thus, we achieved CRISPR-based targeting of non-coding regulatory regions in human $\beta$-cells, and our in vivo studies of a putative 
A

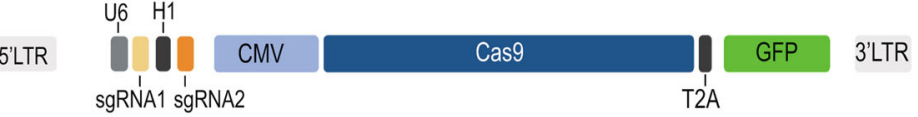

B
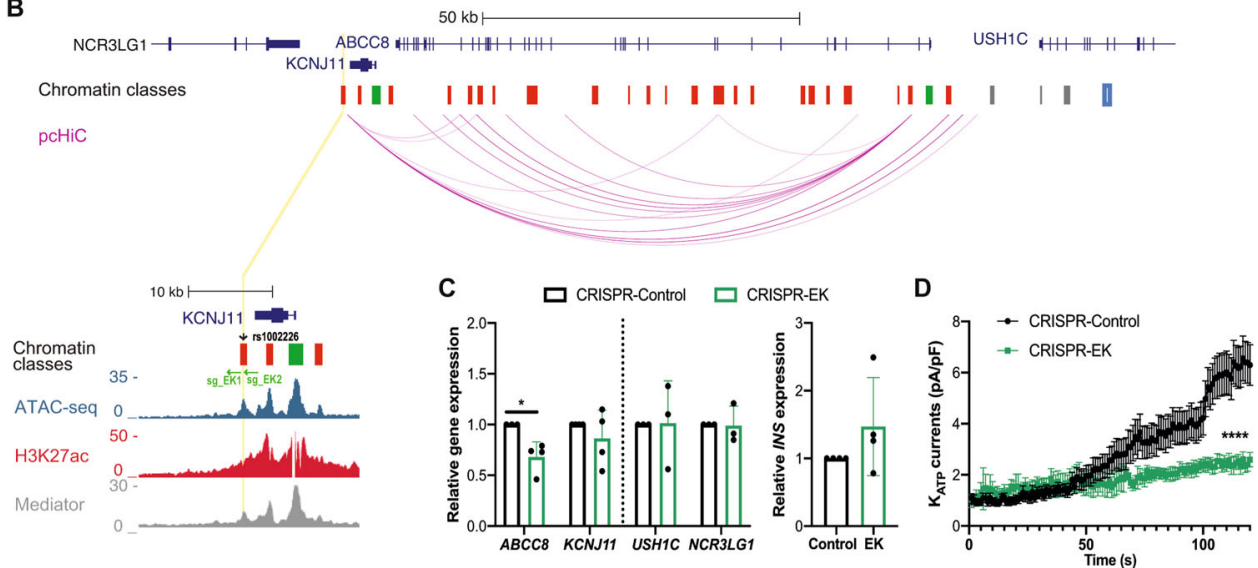

Fig. 4 CRISPR/Cas9 targeting of a non-coding variant in the ABCC8-KCNJ11 locus impairs ABCC8 expression and function in primary human islets. A Schematic of the lenti-construct used for simultaneous expression of two sgRNAs, Cas 9 and GFP (12,021 bp) in primary human islets. B Genome Browser tracks of the genomic context in the KCNJ11-ABCC8 locus, arcs representing high-confidence pcHi-C interactions in human islets, highlighting variant rs1002226 (chr11:17405617) associated with diabetes risk (black arrowhead): This variant maps to a CTCF site (blue) on a class I active enhancer (yellow line, and zoomed inset); sg_EK1 and sg_EK2 flanking rs1002226 (green arrows); Chromatin classes: active promoter (green); active enhancer (red); inactive enhancer (gray); inactive open chromatin (black); strong CTCF (blue). Accessible chromatin regions in human islets are shown by ATAC-seq, H3K27ac, and Mediator ChiP-seq. C ABCC8 mRNA is regulated by the rs1002226-containing enhancer in GFP+ pseudoislet cells, CRISPR-EK (green), CRISPR-Control (Black) $(P=0.0232 ; n=4)$, while expression of $K C N J 11$, control genes (USH1C, NCR3LG1) in the same transcription activation domain, TAD $(n=3)$ and insulin $(n=4)$ were not modified. Data are presented as mean values +SD. D Measurement of KATP currents in GFP+ islet cells from CRISPREK $(n=26$ cells, 3 replicates $)$ and CRISPR-Control $(n=33,3$ replicates $)(P<0.0001)$. Data are presented as mean values \pm SEM. Two-tailed t tests were used to generate $P$-values. ${ }^{\star} P<0.05,{ }^{\star \star \star \star} P<0.0001$. Source data are provided as a Source Data file.

enhancer in native $\beta$-cells revealed a selective impact on the expression of $A B C C 8$ compared to KCNJ11.

LentiCRISPR-pseudoislet based methods could provide a powerful strategy for in vivo analysis of the regulation of genes that are active in human islets but not in other experimental models ${ }^{10}$. To assess this possibility, we targeted the SIX2-SIX3 locus, which encodes the transcription factors SIX2 and SIX3, and the SIX3 antisense (SIX3-AS1) lncRNA ${ }^{10}$, that are expressed in human islet $\beta$-cells, but not in human cell lines, $\alpha$-cells, or in mouse islets ${ }^{10,11,40}$. Nucleotide variants in the locus encoding SIX2 and SIX3 (Fig. 5A) have been linked by GWAS to increased risk for type 2 diabetes (T2D) and diabetes-related traits ${ }^{41-44}$. These variants cluster in a putative SIX2-SIX3 enhancer element previously assessed with episomal luciferase assays in transgenic immortalized mouse $\beta$-cell lines ${ }^{45}$ (SIXE: Fig. $5 \mathrm{~A}$ ), but not in native human $\beta$-cells. We performed cis-expression quantitative trait loci (eQTL) mapping in 292 human islet samples (Methods), and found two variants within this putative enhancer, rs12712929 and rs12712928, showing strong association with islet eQTLs for SIX2 and SIX3, in agreement with previously reported associations ${ }^{45}$. SIX3 expression level was reduced in pancreatic islets with variant $\mathrm{rs} 12712929-\mathrm{TT}(\mathrm{q}-\mathrm{val}=4.9 \mathrm{e}-12)$, while reduced islet expression of SIX2 was strongly associated with rs12712928 -CC (q-val=0.008: Fig. 5B, Supplementary Fig. 5A). However, our recent chromatin conformation studies of this locus $^{35}$, including virtual 4C mapping (Supplementary Fig. 5B), did not detect high-confidence interactions between this SIXE element and SIX2 or SIX3 promoter regions. Thus, the in vivo genetic targets of the SIXE element remain to be established.

To evaluate this putative regulatory element in native $\beta$-cells, we transduced primary human islets with lentivirus (Methods) encoding Cas9, GFP, and two sgRNAs targeting enhancer sequences located $5^{\prime}\left(\right.$ SIXE- $\left.5^{\prime}\right)$ and $3^{\prime}$ (SIXE- $3^{\prime}$ ) of the SNP cluster
(Fig. 5C). After transduction and pseudoislet formation, we performed ddPCR on $\mathrm{GFP}^{+}$cells (Methods; $n=2$ independent donors) and quantified the specificity and extent of mutations induced with these sgRNAs. Transduction with SIXE- $5^{\prime}$ led to indels at the site targeted by SIXE- $5^{\prime}$ but not by SIXE- $3^{\prime}$ (Supplementary Fig. 6A). Likewise, SIXE- $3^{\prime}$ transduction produced indels only at the site targeted by SIXE- $3^{\prime}$ and not SIXE- $5^{\prime}$ (Supplementary Fig. 6B). Transduction with both SIXE-5 $5^{\prime}$ and SIXE- $3^{\prime}$ led to an average $48 \%$ mutation frequency, including indels at the site targeted by sgRNAS SIXE- $5^{\prime}(22 \%)$, at the site targeted by SIXE-3' (10\%), or deletions between these sites (16\%) (Fig. 5C-G). Measures of mRNA using qRT-PCR in purified GFP + cells targeted with the lentiCRISPR-SIXE- $5^{\prime}-3^{\prime}$ (Fig. 5H), or SIXE-5' sgRNA alone (Supplementary Fig. 6C) revealed significantly reduced levels of SIX2, SIX3 or SIX3-AS1 mRNA $(n=6$ independent donors). Assessment of the correlation between the degree of SIXE- $5^{\prime}-3^{\prime}$ editing and the expression level of SIX2, SIX3, or SIX3-AS1 suggests that the effect of SIXE targeting varied between human donor samples, as indicated by the range of gene expression changes noted (Supplementary Fig. 6E). Targeting of SIXE with the SIXE-3' sgRNA or the non-targeting sgRNA did not significantly reduce levels of SIX2, SIX3, or SIX3-AS1 (Fig. $5 \mathrm{H}$, Supplementary Fig. 6C). Levels of other mRNAs from genes inside the same TAD, such as CAMKMT and SRBD1, or in adjacent TADs, such as PRKCE, were not affected by the SIXE- $5^{\prime}-3^{\prime}$ targeting (Fig. 5A, I, Supplementary Fig. 6D; $n=4$ independent donors). We noted a modest reduction of average INS mRNA levels after SIXE- $5^{\prime}-3^{\prime}$ targeting (Fig. 5J), and total insulin content from $\mathrm{GFP}^{+}$SIXE- $5^{\prime}-3^{\prime}$ cells was significantly lower compared to those from non-targeting sgRNAs (Fig. 5K). Thus, our CRISPR targeting data provide evidence that a non-coding genomic element functions as an enhancer in the SIX2-SIX3 locus of human $\beta$-cells. Moreover, localized $5^{\prime}$ or $3^{\prime}$ targeting achieved here 
A
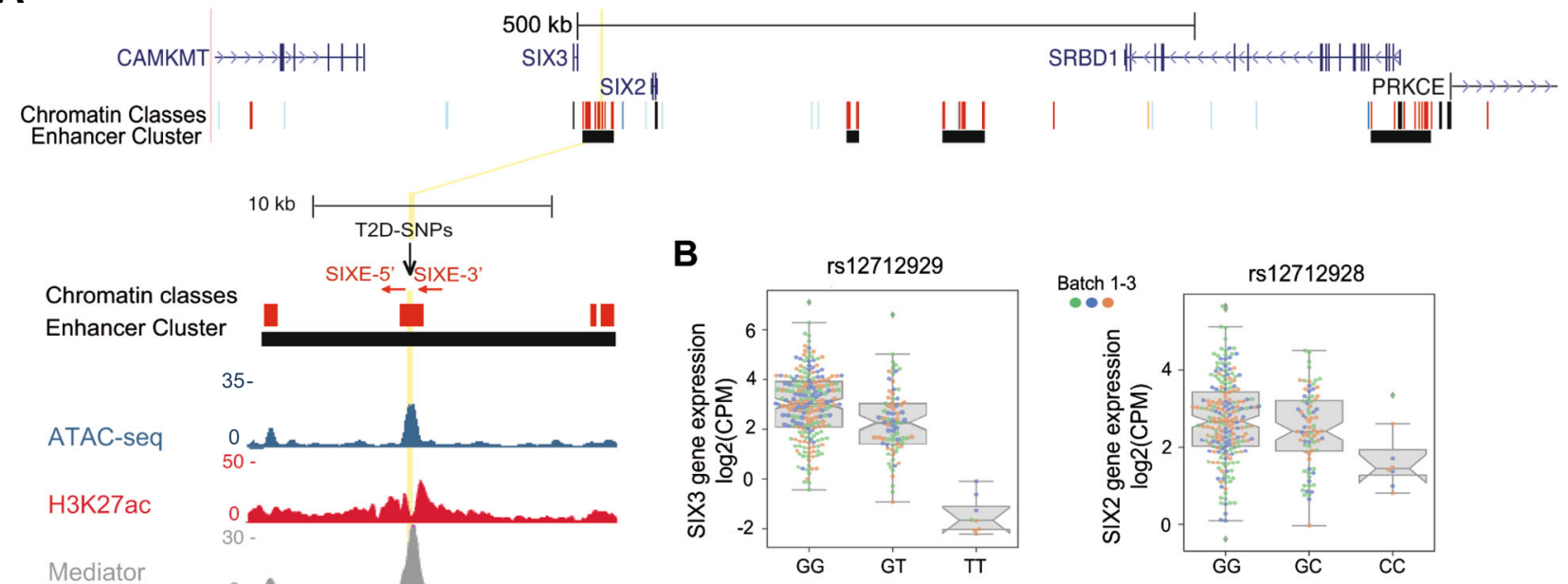

C
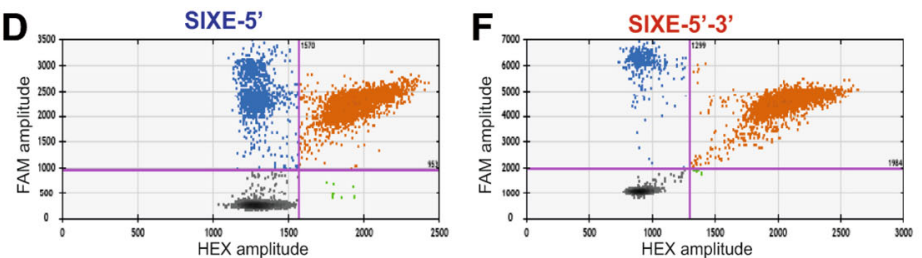

SIXE-3' $\underset{\text { SIX2/SIX3 Enhancer }}{\longrightarrow}$

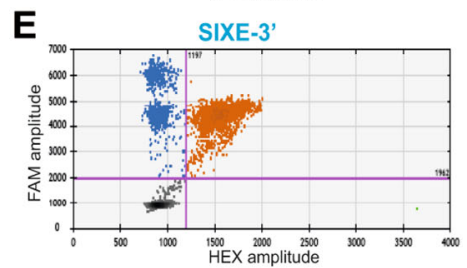

G
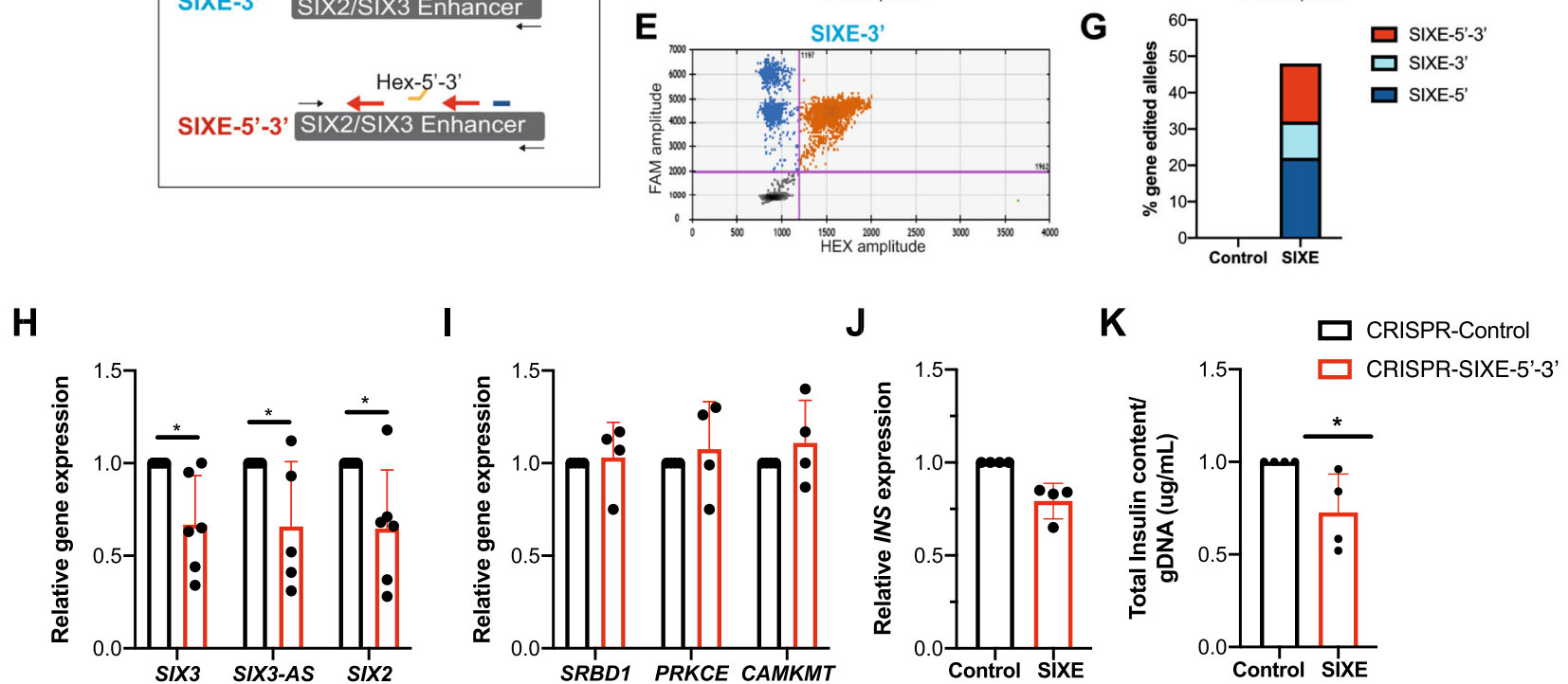

suggested asymmetrical structural features within the SIXE element regulating the expression of SIX2 and SIX3. These findings indicate that in vivo enhancer structure in primary human islet cells can be probed using CRISPR-targeted genomic deletions.

CRISPRa enhancer targeting induces expression of SIX2 and SIX3. In addition to assessing the requirement for the SIXE element in regulating the expression of SIX2 and SIX3, we evaluated the use of CRISPRa to activate this enhancer in primary human islet cells (Fig. 6A). We created a lentiviral construct that simultaneously expresses dCas9-VP64-p65-Rta ${ }^{23}$, a GFP transgene, and two sgRNAs for the SIXE enhancer (SIXE-A1 and SIXE-A2; CRISPRa-SIXE: Methods; Fig. 6B). After infection of primary dispersed human islet cells, pseudoislet development and subsequent purification of $\mathrm{GFP}^{+}$cells (Methods), qRT-PCR revealed increased levels of both SIX2 and SIX3 mRNA after CRISPRa-SIXE compared to controls (Fig. 6C; $n=6$ independent donors); the low abundance of SIX3-AS1 mRNA precluded detection in these studies. Moreover, CRISPRa induction was specific for SIX2 and SIX3: levels of mRNA encoding the neighboring gene PRKCE were indistinguishable in CRISPRa-SIXE and control samples (Fig. 6C). Thus, SIXE enhancer CRISPRa achieved simultaneous induction of SIX2 and SIX3 in native human $\beta$-cells. Consistent with prior studies suggesting that SIX2 regulates INS expression ${ }^{10,22}$, average INS mRNA levels were also modestly increased by CRISPRa-SIXE (Fig. 6D), though this change did not reach statistical significance. Together, our results with CRISPR targeting demonstrate that an enhancer in the SIX2SIX3 locus linked by GWAS to diabetes and diabetes-related traits is necessary and sufficient for in situ regulation of those genes in 
Fig. 5 CRISPR/Cas9 targeting of a putative enhancer element in the SIX2-SIX3 locus in primary human islets. A Genome Browser tracks of the genomic context in the SIX2-SIX3 locus, highlighting the putative SIX2-SIX3 enhancer element (SIXE) with variants previously linked to increased risk of fasting glucose hyperglycemia and T2D (T2D-SNPs, black arrowheads): These variants map to an active enhancer within an enhancer cluster (yellow line, and zoomed inset); sgRNAs used for CRISPR-Cas9 targeting of the region flanking FG-SNP: SIXE-3' and SIXE-5' (red arrows). The two sgRNAs were cloned in the same construct (scheme in Fig. 4A). Chromatin classes: active promoter (green); active enhancer (red); inactive enhancer (gray); inactive open chromatin (black); strong CTCF (blue). Accessible chromatin regions in human islets are shown by ATAC-seq, H3K27ac and mediator ChiP-seq. B Islet eQTLs showing association of reduced expression levels of SIX2 and SIX3 and variants within the SIXE region targeted in this study (cis-eQTL mapping across 292 human islet samples, q-val=4.9e-12 for rs12712929-TT and reduced level of SIX3 and q-val=0.008, for rs12712928 -CC and reduced expression of SIX2). Box plot shows the interquartile range (IQR) of 1st (Q25) and 3rd (Q75) quartiles, with the median as a black line in the center, and whiskers depict \pm 1.5 times the IQR. For SIX3 and GT, GT, and TT genotypes, Q25 values are 2.09, 1.41 and -2.03 , and Q75 values are 3.92, 3.04 , and -1.10 , respectively. For SIX2 and GG, GC and CC genotypes, Q25 values are 2.02, 1.89 and 1.27, and Q75 values are 3.42, 3.20, and 1.03, respectively. C Scheme of the ddPCR assay used to identify gene editing as consequence of targeting by SIXE-5', SIXE-3', or both (SIXE-5'-3') in the pseudoislets targeted with SIXE-5'-3'. HEX probes are shown with an orange line, FAM reference probes, in blue D-F Example of ddPCR $2 \mathrm{D}$ plots showing GFP+ cells modified by (D) SIXE-5', (E) SIXE-3', (F) SIXE-5'-3' (KO cells: FAM ${ }^{+} / \mathrm{HEX}^{-}$, blue droplets; wild-type droplets: FAM ${ }^{+} / \mathrm{HEX}^{+}$, orange, $n=2$ independent donor samples). G Total percentage of gene-edited alleles in GFP+ cells, showing the contribution of (D-F) in the DNA of GFP+ islet cells. H-J mRNA levels of (H) SIX3 ( $n=6$ independent donors; $P=0.0279)$, SIX3-AS1 ( $n=5$ independent donors; $P=0.0437)$ and $S I X 2$ ( $n=6$ independent donors; $P=0.04)$, (I) control genes ( $n=4$ independent donor samples), (J) INS ( $n=4$ independent donor samples) in GFP ${ }^{+}$cells targeted with CRISPR-SIXE-5'-3'. (K) Total insulin content of sorted GFP ${ }^{+}$cells normalized to genomic DNA (gDNA) content $(P=0.0391 ; n=4$ independent donor samples). Data are presented as mean values \pm SD. Two-tailed $t$ tests were used to generate $P$-values. ${ }^{\star} P<0.05$. Source data are provided as a Source Data file.

A

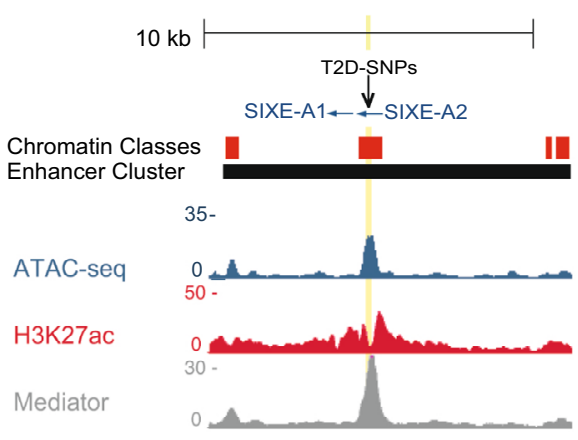

B

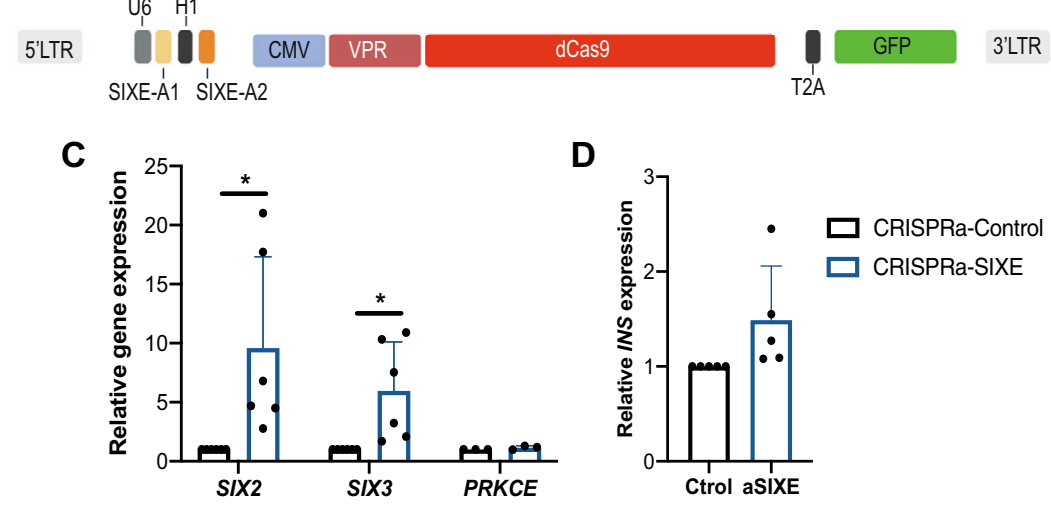

Fig. 6 CRISPR/dCas9 activation of a putative enhancer element in the SIX2-SIX3 locus in primary human islets. A Genome browser tracks highlighting the putative SIX2-SIX3 enhancer element (SIXE) with variants previously linked to increased risk of fasting glucose hyperglycemia and T2D (T2D-SNPS, black arrowheads): These variants map to an active enhancer within an enhancer cluster (yellow line); sgRNAs used for CRISPRa: SIXE-A1, SIXE-A2. Chromatin classes: active enhancer (red); Accessible chromatin regions in human islets are shown by ATAC-seq, H3K27ac, and Mediator ChiP-seq. B Schematics of the lenti-construct used for simultaneous expression of two sgRNAs, VPR, dCas9 and GFP (13,728 bp) in primary human islets. C-D mRNA levels of (C) SIX2 ( $P=0.042 ; n=6$ independent donors), $S I X 3(P=0.0328 ; n=6$ independent donors), PRKCE ( $n=3$ independent donors) and (D) INS ( $n=5$ independent donors), in GFP ${ }^{+}$cells after CRISPRa of SIXE (CRISPRa-SIXE). Data are presented as mean values \pm SD. Two-tailed $t$ tests were used to generate $P$-values. ${ }^{\star} P<0.05$. Source data are provided as a Source Data file.

human $\beta$-cells. In sum, our studies with CRISPR/Cas9 and CRISPRa suggest that the architecture and function of noncoding genomic regions can be interrogated in native human islet cells.

\section{Discussion}

CRISPR/Cas9-based genome editing remains a challenge in quiescent cells (reviewed in ${ }^{46}$ ) and has not been reported in adult pancreatic islet cells, which are post-mitotic ${ }^{16,17}$. Our study reveals index application of CRISPR/Cas9 and CRISPRa for gene editing and activation in primary human islet cells. Our results demonstrate that both coding exons and non-coding regulatory DNA can be mutated in primary human islet cells, resulting in altered gene expression and cellular function. We also demonstrate that targeting regulatory DNA with CRISPRa can induce the expression of genes in primary islet cells. CRISPR-based targeting has been reported in human stem cell-derived insulinproducing cells ${ }^{47}$ and in immortalized $\beta$-cell lines 35,48
(EndoC $\beta H 3$ ). Despite the value of these models, they are fundamentally different from genuine pancreatic islet cells in terms of gene regulation, maturation, function, proliferation, and cellular composition ${ }^{49}$. Thus, the relevance of conclusions from findings with these surrogate cell types to the biology and genetics of human islets remains limited ${ }^{9,10}$. Mutation or activation of regulatory DNA elements with CRISPR in bona fide human islets here achieved selective changes in expression of imputed target genes, and revealed unexpected structural features of islet enhancers. The application of these technologies in primary human islets provides new possibilities to understand genetic regulation of islet biology and disease.

Our findings provide a proof of concept that discrete coding and non-coding DNA elements can be targeted by CRISPR-based approaches in quiescent primary human pancreatic islet cells. In humans, mutations can lead to monogenic forms of diabetes. For example, loss-of-function heterozygous mutations in PDX1 can lead to heritable early-onset diabetes ${ }^{3}$. We created targeted mutation of PDX1 in primary human islet cells, and 
demonstrated for the first time how this could be used to model human islet $\beta$-cell phenotypes resulting from postnatal PDX1 loss. Prior studies in mice suggest that Pdx1 may promote $\beta$-cell survival in some contexts ${ }^{50,51}$, and PDX1 inactivation achieved here could allow future assessment of such roles in adult human $\beta$-cells. For the KCNJ11 locus, we likewise demonstrated that our approach achieved loss-of-function phenotypes in postnatal human islets. Thus, approaches here should be useful for modeling diabetes genetics, including monogenic diabetes, which accounts for approximately $2 \%$ of human diabetes ${ }^{52}$.

CRISPR editing of non-coding DNA elements in human islet $\beta$-cells also provided evidence for the regulation of $A B C C 8$ and the SIX2-SIX3 loci. Deletion within an enhancer element located in the KCNJ11-ABCC8 locus led to reduced expression of one candidate target gene ( $A B C C 8)$ but not the other (KCNJ11, which is more proximal to this enhancer), revealing an unsuspected in vivo function of this enhancer. In another example, CRISPRtargeted deletion in a single putative enhancer led to reduced expression of two nearby genes, SIX2 and SIX3. Thus, CRISPR targeting in primary human islet cells revealed regulatory interactions not previously detected with chromatin conformation assays ${ }^{24}$. This result was corroborated and extended by the finding that sgRNA-based targeting of the $5^{\prime}$ region of the SIXE enhancer, but not the $3^{\prime}$ region, was sufficient to reduce SIX2 and SIX3 mRNA levels, providing evidence in human $\beta$-cells for structural asymmetry in this candidate enhancer. Prior studies ${ }^{53}$ have suggested SIX2 and SIX3 are positioned on opposite sides of a topologically associated domain (TAD), suggesting that regulatory effects of the SIXE enhancer might not extend to genes in an adjacent TAD. Our studies, however, suggest that the SIXE non-coding regulatory element regulates both SIX2 and SIX3. Thus, our work demonstrates that interrogation with CRISPR is a powerful approach for identifying the function of regulatory elements in primary human islets, and is complementary to biochemical or imaging-based studies of chromatin conformation. With further improvements, CRISPR/Cas9 targeting also holds promise for in vivo targeted allele replacement in bona fide native human islet cells. If so, CRISPR/Cas9 targeting in primary islets would provide a consummate experimental means for deconvoluting mechanisms underlying the association of noncoding and coding variants to human type 2 diabetes genetic risk $^{37}$. We speculate that the lentiCRISPR-pseudoislet strategy described here is also applicable to gene editing in human a cells and $\delta$ cells, whose genetic regulation and likely roles in diabetes remain relatively under-explored ${ }^{54,55}$.

\section{Methods \\ Human Islet Procurement. Organs and islets were procured through the Inte- grated Islet Distribution Network (IIDP), National Diabetes Research Institute (NDRI) and the Alberta Diabetes Institute (ADI) Islet Core. De-identified human islets were obtained from healthy, non-diabetic organ donors with less than $18 \mathrm{~h}$ of cold ischemia time, and deceased due to acute trauma or anoxia. For this study, islets from 30 adult donors were used (Supplementary Table 1).}

Constructs and lentivirus production. pRSGCCG-U6-sg-CMV-Cas9-2ATagGFP2 lentiviral constructs coding for Cas9, TagGFP2, and a sgRNA targeting hPDX1, hKCNJ11, or with a non-targeting sequence as control, were obtained from Cellecta Inc. For dual expression of two sgRNAs, pRSGCCG-U6-emptyCMV-Cas9-2A-TagGFP2 was digested with BbsI and XhoI and the scaffold for a second sgRNA was HiFi fused, resulting in pRSGCCG-U6-scaffold-CMV-Cas92A-TagGFP2-dual. The cloning of two sgRNAs and the H1 promoter was performed as previously described ${ }^{39}$. For the generation of an all-in-one-CRISPRadual plasmid, VPR-dCas9 was amplified from pHR SFFV-VPR-dCas9-P2A-Cherry (kindly provided by the Stanley Qi Lab ${ }^{14}$ ) and HIFI fused to amplified pRSGCCGU6-empty-CMV---2A-TagGFP2-dual, resulting in pRSGCCG-U6-empty-CMVVPR-dCas9-2A-TagGFP2-dual. The selection of the enhancer in the KCNJ11$\mathrm{ABCC} 8$ locus was based on findings from pcHi- $\mathrm{C}^{35}$ showing putative associations of this T2D risk variant. The putative enhancer in the SIX2-SIX3 locus was initially described by ${ }^{45}$. The sgRNAs used in this study were designed using E-CRISPR and
MIT CRISPR design tool. All sgRNA sequences used in this study can be found in Supplementary Table 2.

Lentiviruses were produced by transient transfection of HEK293T cells with lentiviral constructs, and with pMD2.G (12259; Addgene) and psPAX2 (12260; Addgene) packaging constructs. Mirus TransIT reagents were used for transfection (Mirus Bio LLC), according to manufacturer recommendations. Supernatants were collected and purified using PEG-it (System Biosciences). Concentrated lentivirus was stored at $-80^{\circ} \mathrm{C}$ until transduction of primary human cells.

Human pseudoislet generation and lentiCRISPR transduction. Human islets were dispersed into a single cell suspension by enzymatic digestion (Accumax, Invitrogen). For each experimental condition, $\sim 1 \times 10^{6}$ cells were transduced with lentivirus corresponding to $1 \times 10^{9}$ viral units in $1 \mathrm{ml}$ as determined by the Lenti-X qRT-PCR titration kit (Clonetech), in medium supplemented with $8 \mathrm{ug} / \mathrm{ml}$ Polybrene. At the time of dispersion and transduction, we exposed cells to the small molecule GNF4877, a DYRK1A inhibitor (MCE: MedChemExpress). Mean transduction efficiencies, determined by $\mathrm{GFP}^{+}$cell quantification, were slightly higher for cells exposed to GNF4877 (2 uM) at the time of infection (41\%) compared to controls (34\%) (Supplementary Fig. 7A). With TIDE PCR, indels were detected in an average of $57.4 \%$ of PDX1 sequences in the absence of GNF4877 (Supplementary Fig. 7B; $n=3$ independent donors). Using ddPCR, 30.2\% mutated alleles were detected for CRISPR-PDX1 without initial exposure to GNF4877 (Supplementary Fig. 7C; $n=2$ independent donors). While prolonged exposure to GNF4877 can induce $\beta$-cell proliferation ${ }^{56}$, its effect alone is not significant ${ }^{57}$. Here, after transient GNF4877 exposure, we did not detect increased production of the proliferation marker MKI67 (Supplementary Fig. 7D). Moreover, reduction of PDX1 mRNA measured by RT-qPCR revealed no difference in outcomes with or without GNF4877 exposure (Supplementary Fig. 7E).

For CRISPRa experiments, TransDux MAX Lentivirus Transduction Reagent was used for infection, per the manufacturer's specifications (System Biosciences). Transduced islet cells were cultured in ultra-low attachment 96-well plates (Corning) for 4 days at $37^{\circ} \mathrm{C}$ with $5 \% \mathrm{CO}_{2}$ in culture medium consisting of RPMI 1640 (Gibco), $2.25 \mathrm{~g} / \mathrm{dl}$ glucose, $1 \%$ penicillin/streptomycin (v/v, Gibco), and $10 \%$ fetal bovine serum (HyClone). On day 4, pseudoislets in all CRISPR/Cas9 experiments were transferred to a 6 well plate in culture medium comprised of RPMI 1640 (Gibco), $2.25 \mathrm{~g} / \mathrm{dl}$ glucose, $1 \%$ penicillin/streptomycin (v/v, Gibco), and $2 \%$ human serum (Sigma-Aldrich), and cultured until day 6 prior to further molecular or physiological analysis. For CRISPRa experiments, pseudoislets were evaluated on day 4 .

FACS sorting. Pseudoislets were dispersed into single cells by brief enzymatic digestion (Accumax, Invitrogen). Cells were stained with LIVE/DEAD ${ }^{\text {mat }}$ Fixable Near-IR Dead Cell Stain Kit (Life Technologies). Non-GFP ${ }^{+}$cells were used as controls. Cells were sorted on a special order 5-laser FACS Aria II (BD Biosciences) using a $100 \mu \mathrm{m}$ nozzle, with doublet removal. Sorted cells were collected into low retention tubes containing $50 \mu \mathrm{L}$ of FACS buffer $(2 \% \mathrm{v} / \mathrm{v}$ fetal bovine serum in phosphate-buffered saline) supplemented with Ribolock RNase inhibitor (Thermo Scientific). DNA, RNA, and/or protein were isolated from sorted $\mathrm{GFP}^{+}$cells. Cytometry data was analyzed and graphed using FlowJo software (TreeStar v.10.8).

Genomic DNA extraction, TIDE, and ddPCR analysis. 1000-5000 $\mathrm{GFP}^{+}$cells were used for genomic DNA extraction using the Arcturus PicoPure $^{\circledast}$ DNA Extraction Kit (Thermo Fisher Scientific) per the manufacturer's instructions. 4 ul of extracted genomic DNA were used as PCR template. PCR was performed with Accuprime Pfx (Thermofisher) in the presence of $4 \mathrm{M}$ betaine (Sigma-Aldrich) in 40 cycles, each cycle composed of a denaturing step at $95^{\circ} \mathrm{C}$ for $30 \mathrm{~s}$, an annealing step at $60^{\circ} \mathrm{C}$ for $40 \mathrm{~s}$ for PDX1, $62^{\circ} \mathrm{C}$ for $1 \mathrm{~min}$ for KCNJ11, and an extension step at $68^{\circ} \mathrm{C}$ for $30 \mathrm{~s}$, followed by a final extension at $68^{\circ} \mathrm{C}$ for $5 \mathrm{~min}$. PCR amplicons were purified with DNA Clean and Concentrator Kit (Zymo Research, Irvine, CA) for sequencing and subsequent TIDE analyses to assess indel efficiency. For ddPCR, a whole-genome amplification step using Repli-g midi kit (Qiagen), was performed. QX200 ddPCR machine (Bio-Rad) was used following the manufacturer's specifications. Data were analyzed using QuantaSoft Software (Bio-Rad). Primer and probes sequences are summarized in Supplementary Table 3.

Evaluation of CRISPR/Cas9 off-target effects; RNA extraction, quantitative RT-PCR. Off-target site prediction was performed using the CHOP-CHOP tool ${ }^{33}$ The potential off-target sites found had at least 2 mismatches with respect to the sgRNA sequence. PCR primers encompassing 4 of these potential off-target sites were designed and the PCR amplicons were purified and sequenced. PCR was performed with Accuprime Pfx (Thermofisher) in the presence of $4 \mathrm{M}$ betaine (Sigma-Aldrich) in 35 cycles, each cycle composed of a denaturing step at $95^{\circ} \mathrm{C}$ for $1 \mathrm{~min}$, an annealing step at $60^{\circ} \mathrm{C}$ for $1 \mathrm{~min}$ and an extension step at $68^{\circ} \mathrm{C}$ for $1 \mathrm{~min}$, followed by a final extension at $68^{\circ} \mathrm{C}$ for $5 \mathrm{~min}$. Primer sequences are summarized in Supplementary Table 3.

RNA was isolated from $\mathrm{GFP}^{+}$pseudoislet cells using the PicoPure RNA Isolation Kit (Life Technologies). cDNA was synthesized using the Maxima First Strand cDNA synthesis kit (Thermo Scientific) and gene expression was assessed by PCR using the Taqman Gene Expression Mix (Thermo Scientific). Low recovery of mRNA from 
some pseudoislet preparations after PDX1-CRISPR precluded assessment of some gene expression. Data were analyzed using Prism 6.0 h (GraphPad Software Inc., San Diego, CA). Paired two-tailed t tests were used to indicate statistical significance, and data are presented as mean and standard deviation.

\section{Perifusion, insulin, and glucagon content measurement, and Immunohis-} tochemistry. Human islet perifusion experiments were performed using a 4 chamber Biorep Perifusion System following the manufacturer's instructions. Equal batches of 100 human pseudoislets from CRISPR-Control and CRISPR-PDX1 from three independent donors were perifused at a flow rate of $100 \mathrm{ul} / \mathrm{min}$ for a total of $142 \mathrm{~min}$ in perifusion buffer with $5.6 \mathrm{mM}, 16.7 \mathrm{mM}$ and $16.7 \mathrm{mM}+\mathrm{IBMX}$ glucose concentrations. High glucose $(16.7 \mathrm{mM})$ stimuli were applied for $30 \mathrm{~min}$ and glucose $(16.7 \mathrm{mM})+$ IBMX was applied for $12 \mathrm{~min}$. Fractions of perifusate were collected every $3 \mathrm{~min}$. The first 10 fractions of each experiment were used to equilibrate islets in the perifusion system and discarded (30 min wash). For each experiment, a total of 30 fractions per condition were collected.

To determine total cellular insulin or glucagon content, both intact transduced pseudoislets ( $n=15$ pseudoislets, 4 independent donors) and sorted GFP ${ }^{+}$cells (4000 cells, 3 independent donors) from CRISPR-PDX1 versus CRISPR-Control pseudoislets and sorted $\mathrm{GFP}^{+}$cells (4000 cells, 3 independent donors) from CRISPRSIXE versus CRISPR-Control pseudoislets were sonicated and lysed to extract the total cellular insulin or glucagon content (Human insulin and glucagon ELISA kits, Mercodia). Data were analyzed using Prism 6.0 h (GraphPad Software Inc., San Diego, CA), normalized to the CRISPR-Control and presented as mean with standard deviation. Paired two-tailed $t$ tests were used to indicate statistical significance.

For immunostaining, human pseudoislets were fixed for $1 \mathrm{~h}$ at $4{ }^{\circ} \mathrm{C}$ and embedded in collagen (Wako Chemicals); mouse pancreata were fixed in $4 \%$ paraformaldehyde overnight at $4{ }^{\circ} \mathrm{C}$. Ten micrometers thick frozen sections were cut and stained following standard cryostaining protocols. Briefly, sections were washed in PBS, incubated with blocking solution followed by incubation in permeabilization/blocking buffer ( $1 \%$ bovine serum albumin, $0.2 \%$ non-fat milk, $0.5 \%$ Triton-X in PBS) for $1 \mathrm{~h}$. Primary antibodies were mixed with permeabilization/blocking buffer and incubated at $4{ }^{\circ} \mathrm{C}$ overnight. The following primary antibodies were used: goat anti-PDX1, (1:200; Sigma-Aldrich 06-1385), Guinea pig anti-insulin (1:500, a0564 Dako), mouse anti-glucagon (1:500, G2654 Sigma), Alexa Fluor 555 Goat anti Guinea Pig IgG $(H+$ L) Secondary Antibody (A21435; Invitrogen) (1:500, Invitrogen), Alexa Fluor ${ }^{\circledR}$ 647-AffiniPure Donkey Anti-Mouse IgG (H + L) (715-605-150; Jackson ImmunoResearch) (1:500). Slides were washed with PBS, incubated with secondary antibodies at room temperature for $2 \mathrm{~h}$, and were preserved with mounting medium containing DAPI (Vector Labs, Vectashield H-1200). Images were obtained using a Leica SP2 confocal microscope.

\section{Transplantation and in vivo assessment of pseudoislet function. Human} pseudoislets were transduced and cultured as described above. Batches of 300 pseudoislets were resuspended in cold Matrigel and transferred into the left renal capsular space of host animals using a glass micro-capillary tube. Transplant recipients were 8-week-old male NOD scid IL2R $\gamma$ null mice (stock number 005557; The Jackson Laboratory) and were anesthetized using ketamine/xylazine. Appropriate depth of anesthesia was confirmed by lack of toe-pinch response. Onemonth post-transplantation, mice were administered an intraperitoneal glucose injection at a dosage of $3 \mathrm{~g} / \mathrm{kg}$ body weight. Glucose measurements and blood samples were collected via the tail vein at $0,15,30,45,60,120$, and 180 min post glucose injection. Human insulin is distinguishable from mice insulin, which allowed us to measure its levels by a human insulin ELISA kit (Mercodia).

Patch-clamp studies. Single-cell patch-clamp studies were performed as described previously ${ }^{58}$. Briefly, human pancreatic pseudoislets were dissociated to single cells and cultured in low glucose conditions $(5.5 \mathrm{mmol} / \mathrm{L}$ ) for 1-3 days. Before starting whole-cell patch clamping, media was changed to bath solution containing (in $\mathrm{mM}): 118 \mathrm{NaCl}, 20$ Tetraethylammonium-Cl, $5.6 \mathrm{KCl}, 1.2 \mathrm{MgCl} 2,2.6 \mathrm{CaCl} 2,5$ HEPES, and 5 glucose $(\mathrm{pH} 7.4$ with $\mathrm{NaOH})$ in a heated chamber $\left(32-35^{\circ} \mathrm{C}\right)$. For patch-clamping, fire polished thin wall borosilicate pipettes, which were coated with Sylgard (3-5 MOhm), contained intracellular solution with (in mM): $125 \mathrm{Cs}$ glutamate, $10 \mathrm{CsCl}, 10 \mathrm{NaCl}, 1 \mathrm{MgCl} 2,0.05$ EGTA, 5 HEPES, 0.1 cAMP, and 3 MgATP (pH 7.15 with CsOH). Electrophysiological measures were collected using a HEKA EPC10 amplifier and PatchMaster Software (HEKA Instruments Inc, Lambrecht/Pfalz, Germany) within 5 min of break-in. Quality control was assessed by the stability of seal $(>10 \mathrm{GOhm})$ and access resistance $(<15 \mathrm{MOhm})$ over the course of the experiment. Data were analyzed using FitMaster (HEKA Instruments Inc) and Prism 6.0 h (GraphPad Software Inc., San Diego, CA).

Expression quantitative trait loci (eQTLs). To identify genetic effects on islet gene expression, we compiled 191 publicly available genotype and RNA-seq samples ${ }^{59,60}$ (GEO and EGA accession numbers GSE50244 and

EGAS00001001265) and 101 in-house samples from human islet donors without diagnosis of diabetes (after QC analysis). cis-eQTL mapping across 292 human islet samples was performed using QTLtools ${ }^{61}$ and a cis-window of $500 \mathrm{~kb}$ up- and downstream of the transcription start site (TSS). 10 principal components (PCs) derived from gene expression and 4 genetic PCs were used as covariates in the linear model. Genetic PCs and population structure characterization were calculated using flashPCA ${ }^{62}$ on a subset of genotyped SNPs that were common in all 4 cohorts, with $\mathrm{MAF} \geq 1 \%$ and missingness $<5 \%$ across all 292 samples. We also excluded SNPs in high $\mathrm{LD}$ (pairwise $\mathrm{r} 2 \leq 0.1$ within $1 \mathrm{Mb}$ window), $\mathrm{C} / \mathrm{G}$ and $\mathrm{A} / \mathrm{T}$ SNPs to avoid strand mismatches, and SNPs located in previously reported regions with long-range LD. We aggregated 1000 Genomes Phase3 reference dataset using overlapping variants to further characterize population structure. Gene expression PCs were calculated using prcomp on normalized counts. To identify the best associated cis eQTL SNP-eGene pairs, QTLtools was run using the permutation pass mode (1000 permutations), and beta approximated permutation $P$-values were adjusted for multiple testing correction using Storey q-values implemented in the qvalue $\mathrm{R}$ package ${ }^{63}$. The significance threshold was set at $\mathrm{q}$-value $\leq 0.01$.

Study approval. Animal experiments were approved and performed in accordance with the guidelines provided by the Stanford University Institutional Animal Care and Use Committee (IACUC)

Data visualization. Browser tracks were made with the UCSC genome browser. The graphics were made with BioRender.

Reporting summary. Further information on research design is available in the Nature Research Reporting Summary linked to this article.

\section{Data availability}

The data that support the findings of this study are available from the authors on reasonable request, see author contributions for specific data sets. Source data are provided with this paper.

Received: 13 October 2020; Accepted: 22 March 2021; Published online: 23 April 2021

\section{References}

1. Gloyn, A. L. et al. Activating mutations in the gene encoding the ATPsensitive potassium-channel subunit Kir6.2 and permanent neonatal diabetes. N. Engl. J. Med. 350, 1838-1849 (2004).

2. Njølstad, P. R. et al. Neonatal diabetes mellitus due to complete glucokinase deficiency. N. Engl. J. Med. 344, 1588-1592 (2001).

3. Stoffers, D. A., Zinkin, N. T., Stanojevic, V., Clarke, W. L. \& Habener, J. F. Pancreatic agenesis attributable to a single nucleotide deletion in the human IPF1 gene coding sequence. Nat. Genet. 15, 106-110 (1997).

4. Ashcroft, F. M. \& Rorsman, P. Diabetes mellitus and the $\beta$ cell: the last ten years. Cell 148, 1160-1171 (2012).

5. Inshaw, J. R. J., Cutler, A. J., Burren, O. S., Stefana, M. I. \& Todd, J. A. Approaches and advances in the genetic causes of autoimmune disease and their implications. Nat. Immunol. 19, 674-684 (2018).

6. Fuchsberger, C. et al. The genetic architecture of type 2 diabetes. Nature 536 41-47 (2016)

7. Hart, P. A. et al. Type $3 c$ (pancreatogenic) diabetes mellitus secondary to chronic pancreatitis and pancreatic cancer. Lancet Gastroenterol. Hepatol. 1, 226-237 (2016).

8. Pasquali, L. et al. Pancreatic islet enhancer clusters enriched in type 2 diabetes risk-associated variants. Nat. Genet. 46, 136-143 (2014).

9. Sneddon, J. B. et al. Stem cell therapies for treating diabetes: progress and remaining challenges. Cell Stem Cell 22, 810-823 (2018).

10. Arda, H. E. et al. Age-dependent pancreatic gene regulation reveals mechanisms governing human $\beta$ cell function. Cell Metab. 23, 909-920 (2016).

11. Benner, C. et al. The transcriptional landscape of mouse beta cells compared to human beta cells reveals notable species differences in long non-coding RNA and protein-coding gene expression. BMC Genomics 15, 620 (2014).

12. Mali, P. et al. RNA-guided human genome engineering via Cas9. Science 339, 823-826 (2013).

13. Cong, L. et al. Multiplex genome engineering using CRISPR/Cas systems. Science 339, 819-823 (2013)

14. Dominguez, A. A., Lim, W. A. \& Qi, L. S. Beyond editing: repurposing CRISPR-Cas9 for precision genome regulation and interrogation. Nat. Rev. Mol. Cell Biol. 17, 5-15 (2016).

15. Balboa, D., Prasad, R. B., Groop, L. \& Otonkoski, T. Genome editing of human pancreatic beta cell models: problems, possibilities and outlook. Diabetologia 62, 1329-1336 (2019).

16. Teta, M., Long, S. Y., Wartschow, L. M., Rankin, M. M. \& Kushner, J. A. Very slow turnover of beta-cells in aged adult mice. Diabetes 54, 2557-2567 (2005).

17. Meier, J. J. et al. Beta-cell replication is the primary mechanism subserving the postnatal expansion of beta-cell mass in humans. Diabetes 57, 1584-1594 (2008). 
18. Chen, H. et al. Polycomb protein Ezh2 regulates pancreatic $\beta$-cell Ink4a/Arf expression and regeneration in diabetes mellitus. Genes Dev. 23, 975-985 (2009).

19. Akerman, I. et al. Human pancreatic $\beta$ cell lncRNAs control cell-specific regulatory networks. Cell Metab. 25, 400-411 (2017).

20. Peiris, H. et al. Discovering human diabetes-risk gene function with genetics and physiological assays. Nat. Commun. 9, 3855 (2018).

21. Walker, J. T. et al. Integrated human pseudoislet system and microfluidic platform demonstrate differences in GPCR signaling in islet cells. JCI Insight 5, e137017 (2020).

22. Bevacqua, R. J. et al. SIX2 and SIX 3 coordinately regulate functional maturity and fate of human pancreatic $\beta$ cells. Genes Dev. 35, 234-249 (2021).

23. Chavez, A. et al. Highly efficient Cas9-mediated transcriptional programming. Nat. Methods 12, 326-328 (2015).

24. Jonsson, J., Carlsson, L., Edlund, T. \& Edlund, H. Insulin-promoter-factor 1 is required for pancreas development in mice. Nature 371, 606-609 (1994).

25. Offield, M. F. et al. PDX-1 is required for pancreatic outgrowth and differentiation of the rostral duodenum. Development 122, 983-995 (1996).

26. Ahlgren, U., Jonsson, J., Jonsson, L., Simu, K. \& Edlund, H. $\beta$-Cell-specific inactivation of the mouseIpf1/Pdx1 gene results in loss of the $\beta$-cell phenotype and maturity onset diabetes. Genes Dev. 12, 1763-1768 (1998).

27. Brinkman, E. K., Chen, T., Amendola, M. \& van Steensel, B. Easy quantitative assessment of genome editing by sequence trace decomposition. Nucleic Acids Res. 42, e168 (2014).

28. Findlay, S. D., Vincent, K. M., Berman, J. R. \& Postovit, L.-M. A digital PCRbased method for efficient and highly specific screening of genome edited cells. PLOS ONE 11, e0153901 (2016).

29. Mock, U., Hauber, I. \& Fehse, B. Digital PCR to assess gene-editing frequencies (GEF-dPCR) mediated by designer nucleases. Nat. Protoc. 11, 598-615 (2016).

30. Labun, K. et al. CHOPCHOP v3: expanding the CRISPR web toolbox beyond genome editing. Nucleic Acids Res. 47, W171-W174 (2019).

31. Gannon, M. et al. pdx-1 function is specifically required in embryonic $\beta$ cells to generate appropriate numbers of endocrine cell types and maintain glucose homeostasis. Dev. Biol. 314, 406-417 (2008).

32. Gao, T. et al. Pdx1 maintains $\beta$ cell identity and function by repressing an $\alpha$ cell program. Cell Metab. 19, 259-271 (2014).

33. Aguayo-Mazzucato, C. et al. Mafa expression enhances glucose-responsive insulin secretion in neonatal rat beta cells. Diabetologia 54, 583-593 (2011).

34. Mahajan, A. et al. Fine-mapping type 2 diabetes loci to single-variant resolution using high-density imputation and islet-specific epigenome maps. Nat. Genet. 50, 1505-1513 (2018).

35. Miguel-Escalada, I. et al. Human pancreatic islet three-dimensional chromatin architecture provides insights into the genetics of type 2 diabetes. Nat. Genet. 51, 1137-1148 (2019).

36. Sakamoto, Y. et al. SNPs in the KCNJ11-ABCC8 gene locus are associated with type 2 diabetes and blood pressure levels in the Japanese population. J. Hum. Genet. 52, 781-793 (2007).

37. Gaulton, K. J. et al. Genetic fine mapping and genomic annotation defines causal mechanisms at type 2 diabetes susceptibility loci. Nat. Genet. 47, 1415-1425 (2015).

38. Bonàs-Guarch, $\mathrm{S}$. et al. Re-analysis of public genetic data reveals a rare Xchromosomal variant associated with type 2 diabetes. Nat. Commun. 9, 321 (2018).

39. Beucher, A. \& Cebola, I. One-step dual CRISPR/Cas9 guide RNA cloning protocol. Protocol Exchange. https://doi.org/10.21203/rs.2.1831/v1 (2019).

40. Baron, M. et al. A single-cell transcriptomic map of the human and mouse pancreas reveals inter- and intra-cell population structure. Cell Syst. 3, 346-360 (2016). e4.

41. Kim, Y. J. et al. Large-scale genome-wide association studies in East Asians identify new genetic loci influencing metabolic traits. Nat. Genet. 43, 990-995 (2011).

42. Hachiya, T. et al. Genome-wide meta-analysis in Japanese populations identifies novel variants at the TMC6-TMC8 and SIX3-SIX2 loci associated with HbAlc. Sci. Rep. 7, 16147 (2017).

43. Varshney, A. et al. Genetic regulatory signatures underlying islet gene expression and type 2 diabetes. Proc. Natl Acad. Sci. 114, 2301-2306 (2017).

44. Spracklen, C. N. et al. Identification of type 2 diabetes loci in 433,540 East Asian individuals. Nature 582, 240-245 (2020).

45. Spracklen, C. N. et al. Identification and functional analysis of glycemic trait loci in the China Health and Nutrition Survey. PLoS Genet. 14, e1007275 (2018).

46. Hultquist, J. F. et al. CRISPR-Cas9 genome engineering of primary CD4+ $\mathrm{T}$ cells for the interrogation of HIV-host factor interactions. Nat. Protoc. 14, 1-27 (2019).

47. Tiyaboonchai, A. et al. GATA6 plays an important role in the induction of human definitive endoderm, development of the pancreas, and functionality of pancreatic $\beta$ cells. Stem Cell Rep. 8, 589-604 (2017).
48. Grotz, A. K. et al. A CRISPR/Cas9 genome editing pipeline in the EndoC- $\beta \mathrm{H} 1$ cell line to study genes implicated in beta cell function. Wellcome Open Res. 4, 150 (2019).

49. Tremmel, D. M., Mitchell, S. A., Sackett, S. D. \& Odorico, J. S. Mimicking nature-made beta cells: recent advances towards stem cell-derived islets. Curr. Opin. Organ Transplant. 24, 574-581 (2019).

50. Johnson, J. D. et al. Increased islet apoptosis in Pdx1/- mice. J. Clin. Investig 111, 1147-1160 (2003).

51. Fujimoto, K. \& Polonsky, K. S. Pdx1 and other factors that regulate pancreatic $\beta$-cell survival. Diabetes, Obes. Metab. 11, 30-37 (2009).

52. Gloyn, A. L. \& Drucker, D. J. Precision medicine in the management of type 2 diabetes. Lancet Diabetes Endocrinol. 6, 891-900 (2018).

53. Gómez-Marín, C. et al. Evolutionary comparison reveals that diverging CTCF sites are signatures of ancestral topological associating domains borders. Proc. Natl Acad. Sci. USA 112, 7542-7547 (2015).

54. Tellez, K. et al. In vivo studies of glucagon secretion by human islets transplanted in mice. Nat. Metab. 2, 547-557 (2020).

55. Arrojo E Drigo, R. et al. Structural basis for delta cell paracrine regulation in pancreatic islets. Nat. Commun. 10, 3700 (2019).

56. Shen, W. et al. Inhibition of DYRK1A and GSK3B induces human $\beta$-cell proliferation. Nat. Commun. 6, 8372 (2015).

57. Ackeif, C. et al. GLP-1 receptor agonists synergize with DYRK1A inhibitors to potentiate functional human $\beta$ cell regeneration. Sci. Transl. Med. 12, eaaw9996 (2020).

58. Camunas-Soler, J. et al. Patch-seq links single-cell transcriptomes to human islet dysfunction in diabetes. Cell Metab. 31, 1017-1031 (2020). e4.

59. Fadista, J. et al. Global genomic and transcriptomic analysis of human pancreatic islets reveals novel genes influencing glucose metabolism. Proc. Natl Acad. Sci. USA 111, 13924-13929 (2014).

60. van de Bunt, M. et al. Transcript expression data from human islets links regulatory signals from genome-wide association studies for type 2 diabetes and glycemic traits to their downstream effectors. PLoS Genet. 11, e1005694 (2015).

61. Delaneau, O. et al. A complete tool set for molecular QTL discovery and analysis. Nat. Commun. 8, 15452 (2017).

62. Abraham, G., Qiu, Y. \& Inouye, M. FlashPCA2: principal component analysis of Biobank-scale genotype datasets. Bioinformatics 33, 2776-2778 (2017).

63. Storey, J. D. The positive false discovery rate: a Bayesian interpretation and the q-value. Ann. Stat. 31, 2013-2035 (2003).

\section{Acknowledgements}

The authors thank past and current members of the Kim group, especially Drs. Y. Hang S. Park, and A. Ibarra Urizar, for technical advice and support, Dr. C. Chang for his contributions to human islet isolations (SDRC), Dr. A. Gloyn (Stanford University) for encouragement, and Dr. R. Bottino (Allegheny Health Network) for assistance in tissue procurement. We thank Dr. Jocelyn Manning Fox, Mrs. Nancy Smith, and Mr. James Lyon (University of Alberta) for their contributions to human islet isolations. We gratefully acknowledge organ donors and their families, Canadian organ procurement organizations, particularly the Human Organ Procurement and Exchange (HOPE) program and the Trillium Gift of Life Network, and islet procurement through the Alberta Diabetes Institute Islet Core, Integrated Islet Distribution Program (U.S. NIH UC4 DK098085), the National Disease Research Interchange, and the International Institute for the Advancement of Medicine. R.J.B. was supported by a postdoctoral fellowship from JDRF (3-PDF-2018-584-A-N) and is on leave from the Animal Biotechnology Laboratory, Facultad de Agronomía, Universidad de Buenos Aires/INPA CONICET, CABA, Argentina. A.A.D current affiliation: Sana Biotechnology, South San Francisco. Work in the CRG and ICL was funded by the Wellcome Trust (WT101033), Medical Research Council (MR/L02036X/1), European Research Council Advanced Grant (789055), Ministerio de Ciencia e Innovación (RTI2018-095666-B-I00) and Marato TV3 \#201611. Work in the University of Alberta was supported by a Foundation Grant from the Canadian Institutes of Health Research (CIHR: 148451, MacDonald). P.E.M. holds a Canada Research Chair in Islet Biology. Work in the Kim lab was supported by NIH awards (R01 DK107507; R01 DK108817; U01 DK123743 to S.K.K.), and JDRF Northern California Center of Excellence (to S.K.K. and M. Hebrok). Work here was also supported by NIH grant P30 DK116074 (S.K.K.), and by the Stanford Islet Research Core, and Diabetes Genomics and Analysis Core of the Stanford Diabetes Research Center.

\section{Author contributions}

R.J.B. and S.K.K. conceptualized the study and guided the work. Methodology: R.J.B., S.K.K.; Investigation: R.J.B., X.Q., J.Y.L., M.F., K.T., X.G., W.Z.; S.H.S., A.A.D., I.M.E., S.B.G., G.A.; Writing: R.J.B. and S.K.K. wrote the manuscript with input from all coauthors; Visualization: R.J.B.; Supervision: S.K.K.; Funding acquisition: R.J.B, S.K.K.

\section{Competing interests}

The authors declare no competing interests. 


\section{Additional information}

Supplementary information The online version contains supplementary material available at https://doi.org/10.1038/s41467-021-22651-w.

Correspondence and requests for materials should be addressed to S.K.K.

Peer review information Nature Communications thanks Sabine A. Fuchs and the other, anonymous, reviewer(s) for their contribution to the peer review of this work.

Reprints and permission information is available at http://www.nature.com/reprints

Publisher's note Springer Nature remains neutral with regard to jurisdictional claims in published maps and institutional affiliations. (c) (1) Open Access This article is licensed under a Creative Commons Attribution 4.0 International License, which permits use, sharing, adaptation, distribution and reproduction in any medium or format, as long as you give appropriate credit to the original author(s) and the source, provide a link to the Creative Commons license, and indicate if changes were made. The images or other third party material in this article are included in the article's Creative Commons license, unless indicated otherwise in a credit line to the material. If material is not included in the article's Creative Commons license and your intended use is not permitted by statutory regulation or exceeds the permitted use, you will need to obtain permission directly from the copyright holder. To view a copy of this license, visit http://creativecommons.org/ licenses/by/4.0/.

(C) The Author(s) 2021 